\title{
Homozygous deficiency of ubiquitin-ligase ring-finger protein RNF168 mimics the radiosensitivity syndrome of ataxia-telangiectasia
}

\author{
SS Devgan ${ }^{1}$, O Sanal ${ }^{2}$, C Doil ${ }^{3}$, K Nakamura ${ }^{1}$, SA Nahas ${ }^{1}$, K Pettijohn $^{1}$, J Bartek ${ }^{\star, 3,4}$, C Lukas $^{3}$, J Lukas ${ }^{3}$ and RA Gatti ${ }^{\star, 1,5}$
}

\begin{abstract}
Maintaining genomic integrity is critical to avoid life-threatening disorders, such as premature aging, neurodegeneration and cancer. A multiprotein cascade operates at sites of DNA double-strand breaks (DSBs) to recognize, signal and repair damage. RNF168 (ring-finger nuclear factor) contributes to this emerging pathway of several E3 ubiquitin ligases that perform sequential ubiquitylations on damaged chromosomes, chromatin modifications essential for aggregation of repair complexes at the DSB sites. Here, we report the clinical and cellular phenotypes associated with a newly identified homozygous nonsense mutation in the RNF168 gene of a patient with a syndrome mimicking ataxia-telangiectasia. The mutation eliminated both of RNF168's ubiquitin-binding motifs, thus blocking progression of the ubiquitylation cascade and retention of repair proteins including tumor suppressors 53BP1 and BRCA1 at DSB sites, consistent with the observed defective DNA damage checkpoints/repair and pronounced radiosensitivity. Rapid screening for RNF168 pathway deficiency was achieved by scoring patients' lymphoblastoid cells for irradiation-induced nuclear foci containing 53BP1, a robust assay we propose for future diagnostic applications. The formation of radiation-induced DSB repair foci was rescued by ectopic expression of wild-type RNF168 in patient's cells, further causally linking the RNF168 mutation with the pathology. Clinically, this novel syndrome featured ataxia, telangiectasia, elevated alphafetoprotein, immunodeficiency, microcephaly and pulmonary failure and has implications for the differential diagnosis of autosomal recessive ataxias.
\end{abstract}

Cell Death and Differentiation (2011) 18, 1500-1506; doi:10.1038/cdd.2011.18; published online 11 March 2011

The human genome is continuously exposed to free radicals, ionizing radiation and other genotoxic agents that cause DNA double-strand breaks (DSBs). ${ }^{1-3}$ Over one hundred genes are involved in recognition, signaling and repairing DSBs in order to maintain genomic stability and avoid accumulation of disease-predisposing mutations. ${ }^{2}$ At the cellular level, functions of the DNA damage sensors, signal transducers and effectors ultimately modulate the cell-fate decisions under genotoxic stress conditions, including decisions whether to undergo cell death or survive, or possibly take the route toward oncogenic transformation. ${ }^{2-4}$ At the level of the whole organism, mutations in a subset of such genes are known to predispose to diverse types of cancer, neurodegenerative disorders, premature aging and/or immunodeficiency syndromes. ${ }^{2-4}$ On the other hand, the majority of the DNA damage response (DDR) genes are neither completely characterized nor linked to human disease phenotypes. ${ }^{2-5}$

The RNF168 gene is located on chromosome 3q29 and encodes a 571 amino acid protein that is a member of the 'ring-finger nuclear factor' (RNF) family of ubiquitin ligases.
These enzymes modify functional properties of other proteins by covalent attachment of ubiquitin polypeptides, and emerging evidence suggests that this type of modification has a key role in the hierarchically structured genome surveillance pathways, including responses to DSBs. ${ }^{3,6-8}$ Within seconds and minutes after DSB generation, ATM (ataxia-telangiectasia mutated) protein kinase ${ }^{3,4,9}$ phosphorylates histone $\mathrm{H} 2 \mathrm{AX}$ to become $\gamma-\mathrm{H} 2 \mathrm{AX} . \gamma-\mathrm{H} 2 \mathrm{AX}$ acts as a beacon for identifying sites of DNA damage and initiates a cascade of chromatin modulation and DNA repair events by recruiting MDC1 (mediator of DNA damage checkpoint 1). ${ }^{9}$ This is followed by accumulation of two closely related RNF ubiquitin ligases, RNF8 and RNF168, ${ }^{6-13}$ in concert with the functionally related HECT-domain protein HERC2. ${ }^{14}$ These proteins, along with SUMO-ligases PIAS1 and PIAS4 ${ }^{14-17}$ then trigger and/or amplify binding of ubiquitin and SUMO onto histones near the DNA breaks, thereby forming a scaffold for the local accumulation of essential repair factors, including 53BP1 and yet another ubiquitin ligase, the tumor-suppressor BRCA1. ${ }^{3,6-16}$

\footnotetext{
${ }^{1}$ Department of Pathology and Laboratory Medicine, University of California Los Angeles, Los Angeles, CA, USA; ${ }^{2}$ Immunology Division, Hacettepe University Children's Hospital, Ankara, Turkey; ${ }^{3}$ nstitute of Cancer Biology and Centre for Genotoxic Stress Research, Danish Cancer Society, Copenhagen, Denmark; ${ }^{4}$ Institute of Molecular and Translational Medicine, Palacky University, Olomouc, Czech Republic and ${ }^{5}$ Department of Human Genetics, University of California Los Angeles, Los Angeles, CA, USA. *Corresponding authors: J Bartek, Institute of Cancer Biology and Centre for Genotoxic Stress Research, Danish Cancer Society, Strandboulevarden 49, Copenhagen, DK-2100, Denmark. Tel: + 45 35257357; Fax: + 45 35257721; E-mail: jb @ cancer.dk or RA Gatti, Department of Pathology and Laboratory Medicine, David Geffen School of Medicine, University of California-Los Angeles, Los Angeles, CA 90095-1732, USA. Tel/Fax: + 310825 7618; E-mail: rgatti@mednet.ucla.edu Keywords: DNA damage signaling and repair; RNF168 and BRCA1 ubiquitin ligases; ionizing radiation-induced 53BP1 foci; ataxia-telangiectasia mimicking syndrome; alphafetoprotein, microcephaly

Abbreviations: DDR, DNA damage response; DSB, DNA double strand break; 53BP1, p53-binding protein 1; BRCA1, Breast cancer 1; A-T, ataxia telegiectasia; ATM, ataxia telegiectasia mutated; $A O A$, ataxia with oculomotor apraxia; IPF, idiopathic pulmonary fibrosis; RNF, ring finger nuclear factor; MDC1, mediator of DNA damage checkpoint 1; LCL, lymphoblastoid cell; MIU, motif interacting with ubiquitin; IR, ionizing radiation; CNV, copy number variant; AFP, alpha fetoprotein

Received 17.12.10; revised 24.1.11; accepted 27.1.11; Edited by G Melino; published online 11.3.11
} 
Although disruption of this DNA damage-activated ubiquitin ligase cascade undermines genome integrity and cell survival in experimental models, the translational significance of the individual components of this pathway can only be appreciated by identifying patients who either lack or harbor deficient versions of, one of them. Arguably, the best example of pathophysiological impact of a malfunctional component within this cascade are tumors associated with loss-offunction mutations of BRCA1. ${ }^{3,9,14,16}$ Another well-known example is the complex syndrome of ATM deficiency, known as ataxia-telangiectasia (A-T), a life-threatening disease characterized by a combination of developmental defects, including immune deficiency, neurodegeneration, radiosensitivity and enhanced probability to develop cancer. ${ }^{3,17,18}$

Here, we report a novel clinical syndrome, by identification of a patient lacking the RNF168 protein, with a clinical phenotype that mimicks $A-T,{ }^{17-19}$ including mild gait ataxia, ocular telangiectasia, an elevated alphafetoprotein (AFP) and terminal respiratory failure. Importantly, this phenotype resulted from homozygous nonsense mutation in the RNF168 gene that precluded the expression of the two ubiquitin-binding domains, MIU-1 and MIU-2 (motifs interacting with ubiquitin).$^{6,7}$ In the proposed operational model, absence of RNF168 should prevent the retention of repair proteins, such as 53BP1 and BRCA1 at DSB repair sites, thereby causing a repair defect and increased cell death in response to ionizing radiation. Interestingly, the deficiency detected in our present study was found by screening for irradiation (IR)-induced nuclear foci containing not RNF168 but 53BP1, consistent with the model that RNF168 controls the chromatin ubiquitin-binding pathway upstream of 53BP1 and BRCA1.6,7

\section{Results}

A 16-year-old Turkish male (RS66) initially presented with concerns about short stature. His birth history was unremarkable; he had no learning difficulties and had completed secondary school. Family history was negative and consanguinity was denied. Past history was noncontributory. He had no allergies, was not on medications and review of systems was negative. On physical exam, he was diminutive but alert and interactive. His height $(153 \mathrm{~cm}$ per $5 \mathrm{ft})$ and weight (35 kg per $77 \mathrm{lbs}$ ) were below the fifth percentile for age. His head circumference was $49.5 \mathrm{~cm}$ per $19.5 \mathrm{in}$, $>2$ S.D. below the mean for age and sex. Additional findings were conjunctival telangiectasias and a mild wide-based gait. Cranial nerves, reflexes and sensori-motor exam were normal. $\mathrm{He}$ had no neurobehavioral defects and mental status was intact. Congenital malformations were not present; skin exam was normal, and other organ systems were unremarkable. He experienced maxillary sinusitis several times during the following 3-4 years. With the exception of a serum IgA $<21 \mathrm{mg} / \mathrm{dl}$ (normal range $80-350 \mathrm{mg} / \mathrm{dl}$ ) and serum AFP $147 \mathrm{IU} / \mathrm{ml}$ (normal $<5.9 \mathrm{IU} / \mathrm{ml}$ ), laboratory tests were normal, including karyotyping.

Overall, the patient's initial clinical profile, which included mild ataxia, ocular telangiectasia and immunodeficiency, suggested a mild or variant form of $\mathrm{A}-\mathrm{T}$, an autosomal recessive neurodegenerative disease caused by mutations in

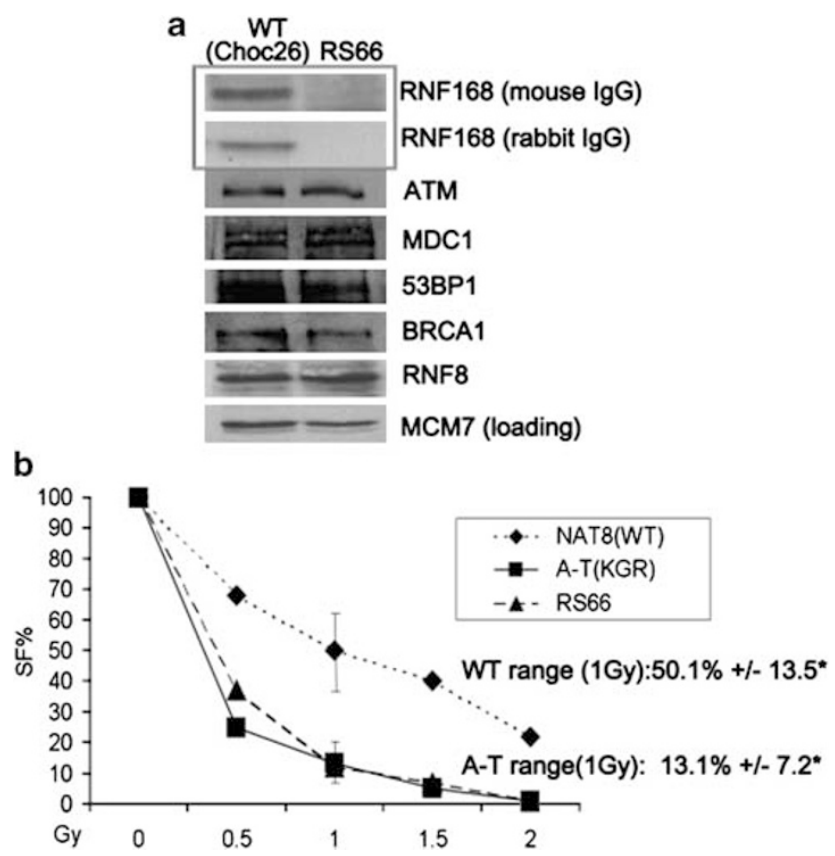

Figure 1 Analysis of protein levels of selected DDR factors and radiosensitivity of RS66 cells. (a) Cell lysates from RS66 and control (Choc26) cells were analyzed by immunoblotting with the indicated antibodies (see Materials and Methods section). The red box highlights the lack of RNF168 protein in RS66 cells. (b) Colony survival assay demonstrates AT-like radiosensitivity of RS66 cells. Asterisk refers to $\mathrm{SF} \%$ ranges defined in Sun et $a .^{20}$ The color reproduction of this figure is available on the html full text version of the manuscript

the ATM gene. ${ }^{17-19}$ In vitro testing of cultured lymphoblastoid cells (LCLs) from RS66 revealed normal amounts of the ATM protein and kinase activity (judged from phosphorylation of several known ATM substrates in response to ionizing radiation), which did not support a conventional diagnosis of A-T (Figure 1a, and data not shown). To rule out unconventional forms of $\mathrm{A}-\mathrm{T}$, the ATM gene was also sequenced; no mutations were identified.

RS66 cells were then tested by colony survival assay, to assess radiosensitivity and repair of DSBs. ${ }^{20,21}$ These cells were poor at forming colonies after IR, with a survival fraction (SF) of $16 \%$ at 1 Gray (Figure $1 \mathrm{~b}$ ); this was within the testing range regarded as 'radiosensitive' (0-21 SF\%, mean SF \pm 1 S.D. of $13.1 \pm 7.2 \%$ ), based on responses of cells from 104 A-T patients and 29 normal controls (see Sun et al. ${ }^{20}$ ). The RS66 cells also exhibited an S-phase checkpoint defect when analyzed for the ability to undergo radioresistant DNA synthesis (Figure 2a), and showed aberrantly persistent phosphorylation kinetics of SMC1-Ser966 in response to radiation (Figure $2 \mathrm{~b}$ ), an ATM-mediated modification of the delayed kinetics that are indicative of impaired DSB repair. In addition, consistent with the aberrantly protracted phosphorylation of SMC1 (Figure 2b), radiation-induced $\gamma-\mathrm{H} 2 \mathrm{AX}$ foci remained abnormally elevated for a longer period of time compared with normal control human LCLs. Specifically, by $1 \mathrm{~h}$ after IR (1 Gy) the percentage of cells with elevated $\gamma-\mathrm{H} 2 \mathrm{AX}$ foci increased from the basal level (below 10\%) to 65, 87 and $64 \%$ in normal control, A-T and RS66 cells, respectively. This was followed by a drop to $14 \%$ in control cells, in contrast to a considerably slower decrease in A-T (to 32\%) and RS66 


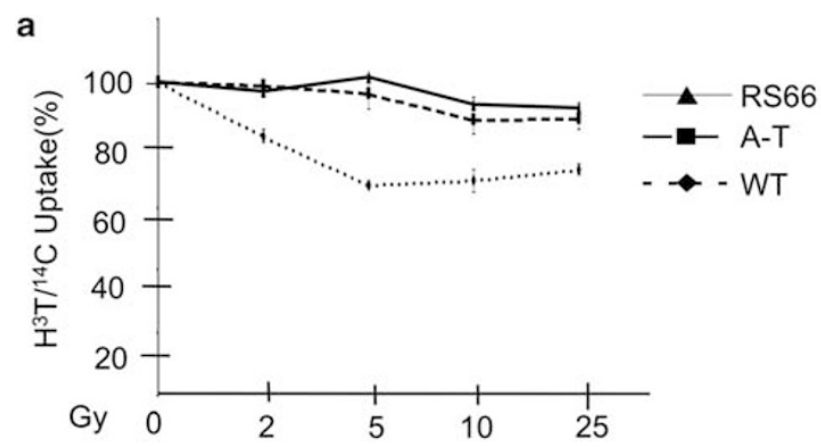

b
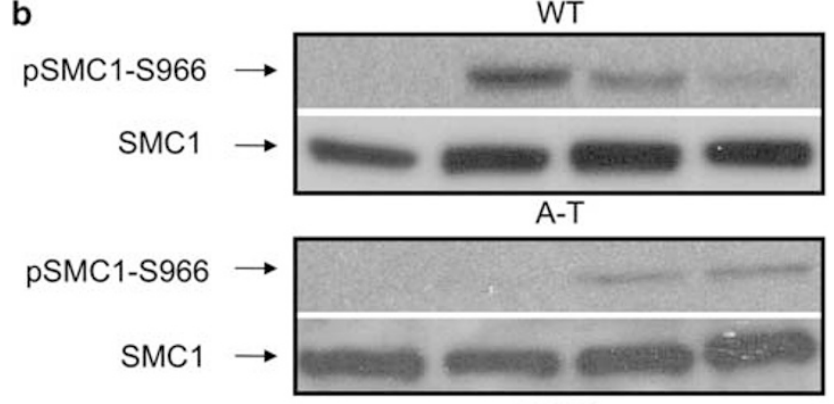

RS66

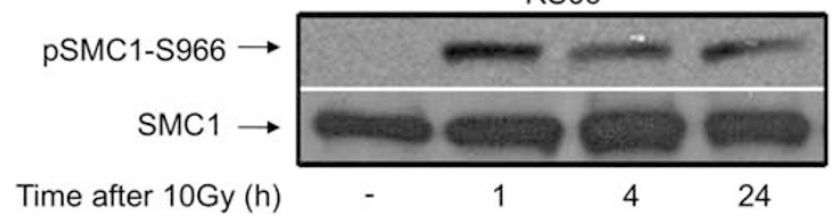

Figure 2 Functional analysis of DDR in RS66 cells. (a) RS66 cells exhibit an intra-S phase cell cycle checkpoint defect similar to A-T cells. WT, A-T and RS66 LCLs were labeled with ${ }^{14} \mathrm{C}$ thymidine for $24 \mathrm{~h}$, irradiated with the doses indicated, and then chased with ${ }^{3} \mathrm{H}$ thymidine $\left(\mathrm{H}^{3} \mathrm{~T}\right)$ for $1 \mathrm{~h}$. Cells were fixed and the $\mathrm{H}^{3} \mathrm{~T} /{ }^{14} \mathrm{C}$ ratio, indicative of S-phase inhibition, was calculated by using a scintillation counter. Error bars represent the mean \pm S.D. $N=3$. (b) RS66 cells maintain increased levels of phosphorylated SMC1s966 $24 \mathrm{~h}$ post-IR. RS66 LCLs were mock-irradiated or exposed to $10 \mathrm{~Gy}$ of IR and fixed at the times indicated after IR (bottom). WT (top) and A-T (middle) LCLs controls were included in each experiment. Nuclear extracts were prepared and subjected to SDS-PAGE/western blot analysis using the antibodies indicated

(to $41 \%$ ) cells, respectively, by $24 \mathrm{~h}$ after radiation. Thus, the kinetics of recovery after IR, as monitored by persistence of $\gamma-\mathrm{H} 2 \mathrm{AX}$ foci, indicates a cellular DSB repair defect in RS66 cells comparable to ATM deficiency. On the other hand, results of mitochondrial respiration measured in RS66 cells (assessed by a resazurin assay ${ }^{22}$ ), and the enzymatic machinery used for DNA end joining (assessed by an in vitro plasmid-based assay, ${ }^{23}$ in RS66 cell extracts) were within the normal range (negative data, not shown).

The patient was subsequently lost to follow-up until age 29 when he was admitted with chronic shortness of breath and cough. He reported having chronic shortness of breath for the previous 3 years and non-productive cough for the previous 5 years. He smoked (1/2 pack per day) from 19 to 23 years of age. He had no history of toxic exposures, chronic pulmonary infections, sarcoidosis, connective tissue diseases or autoimmune diseases. His skin was very dry (xerosis). He worked in a cafeteria and was married, with one daughter. His height and head circumference were unchanged since age 16 . His weight had increased to $39 \mathrm{~kg}$ (86 lbs). He was intermittently hospitalized with worsening pulmonary function, reduced lung capacity, progressive dyspnea and hypoxemia. By age 28 , he had become oxygen dependent. Pulmonary function tests indicated restrictive pathology with FEV1/FVC 100\% (FEV1 $34-22 \%$ and FVC $31-20 \%$ ) and a pO2 of $69-43 \mathrm{~mm} \mathrm{Hg}$. Thoracic CAT scans revealed peribronchovascular reticular densities, particularly in the upper and middle lobes, intralobular and interlobular septal thickenings, subpleural interstitial and bilateral focal pleural thickenings, irregular thickenings of major fissures, and traction bronchiectasis. Bronchoscopy revealed increased vascularization of the mucosa of both bronchi, with telangiectasias on the right main bronchus. His ataxia was slightly worse than when seen the last time; he never developed apraxia or dysarthria. At age 30, RS66 died from respiratory failure secondary to idiopathic interstitial pneumonia and presumed pulmonary fibrosis.

Given the A-T-like clinical features of the RS66 patient yet wild-type ATM gene and normal expression of ATM protein in the RS66 cells (Figure 1a), we argued that a defect of some other component of the DSB-signaling pathway might underlie the observed pathology and cellular radiosensitivity. In addition, we hypothesized that analogous DSB signaling and/or repair defects might also explain additional cases of radiosensitivity. To search for such potential radiosensitizing molecular defects, we next screened a panel of 80 radiosensitive LCLS of undetermined etiology (i.e., excluding known DNA DSB repair defects associated with radiosensitivity, such as A-T and Nijmegen Breakage Syndrome (NBS)) that also included the RS66 cells. For this focused phenotypic screening, we exploited the recent findings of 53BP1 accumulation at sites of double-strand DNA breaks. ${ }^{6,7,24-26}$ Microscopically, we searched for 53BP1-containing IR-induced nuclear foci as a reliable surrogate marker for integrity of the ubiquitin ligase-regulated DNA repair pathway. ${ }^{6-16}$ Specifically, we exposed the cells to $3 \mathrm{~Gy} I \mathrm{R}$ and $1 \mathrm{~h}$ later coimmunostained the cells with antibodies to 53BP1 and $\gamma \mathrm{H} 2 \mathrm{AX}$ or MDC1 (the antibodies to $\gamma \mathrm{H} 2 \mathrm{AX}$ and MDC1 served as unbiased indicators of DSB damage). Strikingly, RS66 was the only one of the 80 radiosensitive cell lines that failed to form 53BP1 nuclear foci (Figure 3a). Consistent with this finding, IRinduced BRCA1 foci, which also require local chromatin ubiquitinylation, ${ }^{6-9}$ were also absent in RS66 cells (Figure $3 \mathrm{~b}$ ).

To elucidate the cause of the observed defects in recruitment of the DNA repair proteins at sites of DNA damage, we analyzed protein extracts from control and RS66 cells by western blotting with a panel of antibodies to the key chromatin-associated DSB regulators ${ }^{3,6-9,16}$ (Figure 1a). RNF168 protein was completely absent in RS66 cell lysates. Since only one antibody to RNF168 was available at the time of these studies, we also raised a new, mouse monoclonal antibody to RNF168 (DCS-402) to validate the lack of the protein in RS66 cells. As shown in Figure 1a, the absence of RNF168 was reproducible and confirmed by the two independent antibodies to RNF168, suggesting that the lack of this factor might be the molecular explanation for the inability of RS66 cells to retain the 53BP1 and BRCA1 repair proteins around DNA repair break sites. Interestingly, immunoblotting also confirmed that other components of this DSB signaling and repair pathway, such as MDC1, 53BP1, 
a

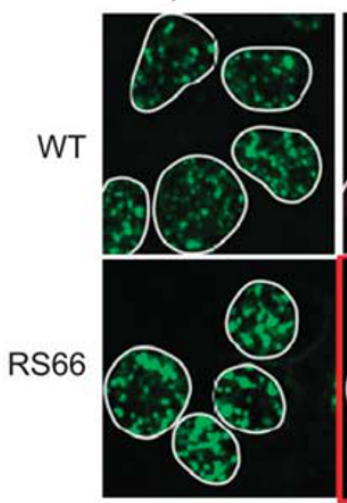

b

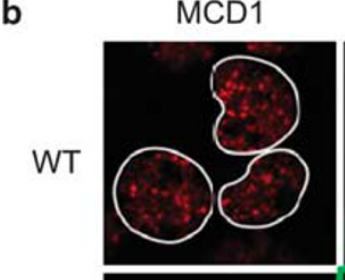

RS66

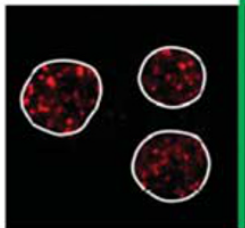

53BP1
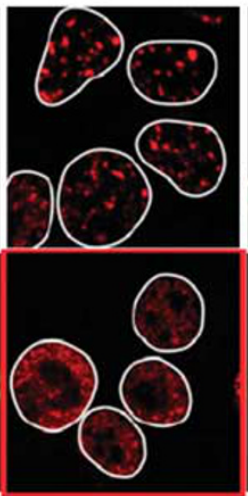

BRCA1
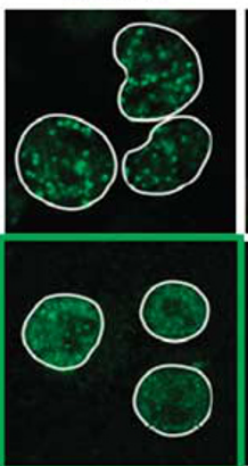

merge

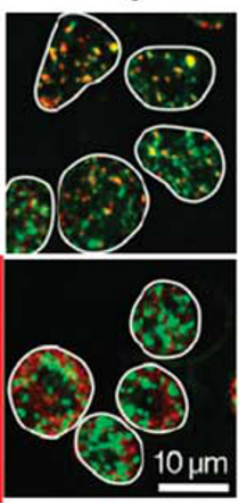

merge
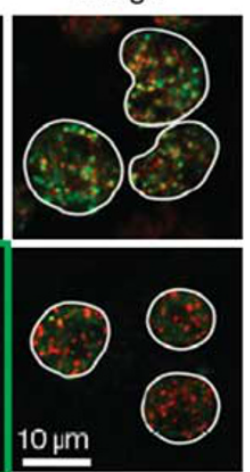

Figure 3 Immunocytochemical analysis of 53BP1 and BRCA1 foci formation in response to ionizing radiation. (a) 53BP1 IRIF formation. RS66 and control (Choc26) cells were exposed to 3 Gy of IR and $1 \mathrm{~h}$ later immunostained with the antibodies to 53BP1 and gamma-H2AX. The red-framed field shows cells with impaired focal accumulation of 53BP1. (b) BRCA1 IRIF formation. RS66 and control (Choc26) cells were treated as in (a) and immunostained with antibodies to BRCA1 and MDC1. The green-framed field shows cells with impaired focal accumulation of BRCA1

BRCA1 and RNF8, were present in normal amounts in RS66 cells (Figure 1a).

DNA sequencing of the entire RNF168 gene identified two homozygous nonsense mutations, $391 \mathrm{C}>\mathrm{T}$, in exon 3 (Figure 4a). These mutations are predicted to create a premature stop codon during translation of the RNF168 protein that would eliminate the key structural domains of the mature protein required for its function as a ubiquitin ligase, MIU-1 and MIU-2. ${ }^{6-8}$ The parents of the RS66 patient were no longer alive for follow-up sequencing.

Although the above results strongly implicated RNF168 in $\mathrm{RS66}$-associated DNA repair, it was possible that mutations in other genes might cause the disease phenotype. Such possibility was excluded by genetic complementation of RS66 cells, whereby reintroduction of a full-length RNF168 cDNA construct that was tagged with green fluorescent protein $(\mathrm{GFP})^{6}$ robustly restored both $53 \mathrm{BP} 1$ and BRCA1 retention in IR-induced nuclear foci (Figure $4 b$ ).

\section{Discussion}

Collectively, our analyses provide evidence that the absence of the RNF168 protein and its associated catalytic activity prevent retention of 53BP1 and BRCA1 proteins at sites of DSB repair, in accordance with the recently proposed model, ${ }^{5-7}$ and thereby are the major cause of the DSB repair malfunction in RS66 cells. Our present data further indicate that insufficient repair of DNA breaks translates into the pathophysiological phenotype observed in this patient, thereby identifying a novel syndrome that resembles the clinical features and cellular phenotypes associated with A-T.

RS66 succumbed to respiratory failure at an early age. His clinical course was accompanied by ataxia, growth retardation, microcephaly, immunodeficiency and an elevated serum AFP. An elevated serum AFP has only been described in two other inherited ataxias: A-T and AOA2 (ataxia with oculomotor apraxia type 2) (a.k.a. senataxin deficiency). ${ }^{18,27}$ Elevated AFP is also found in gastric cancers and in the amniotic fluid of fetuses carrying open neural tube defects. However, the pattern of differential glycosylation for the elevated AFP of A-T patients resembles that of hepatic not fetal, gastric or neural origin. ${ }^{19}$ The mechanism for an elevated serum AFP is unclear for any of these conditions. The absence of apraxia and dysarthria would not have supported a diagnosis of A-T or AOA2, although this patient was followed for many years as an 'A-T variant', a term that is often used by clinicians as an operational working diagnosis when no other is more specific. Similarly, a diagnosis of $\mathrm{NBS}^{5}$ would not have been supported by the normal intelligence and the presence of an abundant level of nibrin (NBS1/NBN) protein observed on immunoblots.

Genetic causes of idiopathic pulmonary fibrosis (IPF) remain unclear although surfactant and interleukin genes have been linked to a few familial cases. ${ }^{28-30}$ Accumulation of DNA fragments can trigger an inflammatory cascade. ${ }^{1,28}$ Thus, the respiratory failure and suggested pulmonary fibrosis in this patient may have been caused by chronic inflammation from unrepaired DNA damage. Further clarification of RNF168 function(s) may expand our understanding of IPF. Alternatively, it is possible that the progressive and fatal respiratory failure observed in this single patient may have had another etiology. Severe lymphocytic infiltration with pulmonary failure is often reported in the later stages of $A-T^{31}$ (Table 1).

We show that patient RS66 carried homozygous nonsense mutations in the RNF168 gene, which abolished production of RNF168 protein. Interestingly, the only other patient with RNF168 deficiency (15-9BI) reported to date was heterozygous for two nonsense mutations that both truncated the protein but left one ubiquitin-interacting motif (MIU1) to possibly continue the formation of chromatin ubiquitinylation. ${ }^{7,26}$ In contrast to RS66, this patient did not manifest ataxia, telangiectasia, microcephaly, nor respiratory failure. In common were growth retardation, serum IgA deficiency and radiosensitivity (Table 1 ). No mention was made of AFP. ${ }^{1,26}$ Of great translational interest, both patients were lacking IR-induced 53BP1 and BRCA1 nuclear foci (i.e., the ability to localize and retain these repair factors at the DSB sites was lost). This strongly supports the critical role of the RNF8-RNF168-HERC2-BRCA1 chromatin ubiquitin ligase cascade 8,16 in the maintenance of genome integrity.

Considering the two patients together, RNF168 deficiency may provide insights into the pathogenesis of $\lg A$ deficiency and common variable immune deficiency, which together 


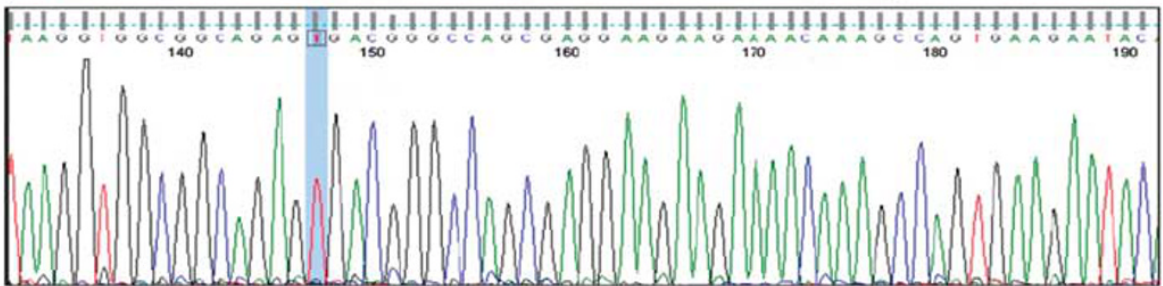

b
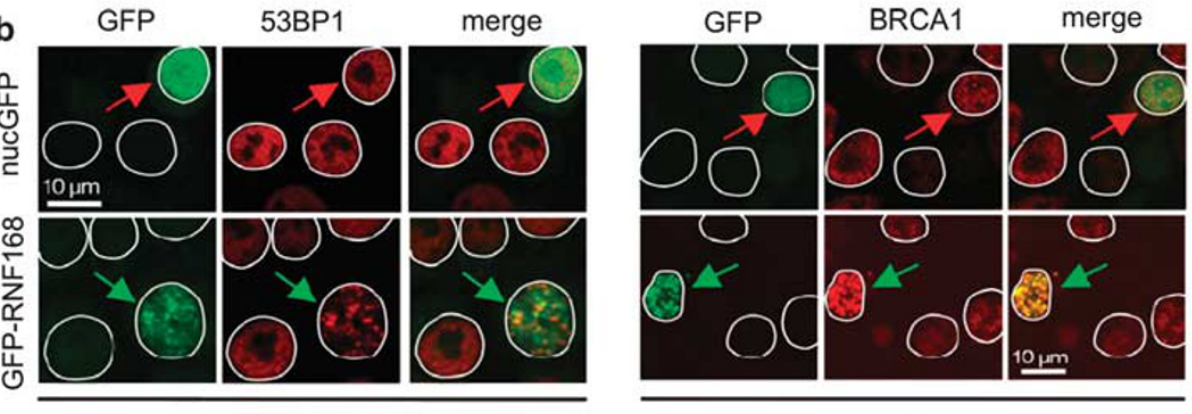

RS66

\section{RS66}

Figure 4 Identification of the RNF168 gene mutation in RS66, and phenotypic rescue of DNA damage signaling by wild-type RNF168 in the RS66 cells. (a) Sequence of genomic DNA from RS66 cells, showing a homozygous nonsense mutation at c.391C > T (R131X) (shaded column). (b) 53BP1 and BRCA1 foci formation in RS66 cells reconstituted by ectopic wild-type RNF168. RS66 cells were transfected with the control plasmid coding for free nuclear GFP (nucGFP, upper row) or the expression plasmid for wild-type GFP-RNF168 (bottom row). The productively transfected cells were identified according to the nuclear GFP signal (green channel) and scored for 53BP1 (left panel) or BRCA1 (right panel) focus formation. Red arrows (upper row) point to control cells lacking nuclear foci for either 53BP1 or BRCA1; green arrows (lower row) mark RNF168-complemented cells that regained the ability to accumulate 53BP1 and BRCA1 in nuclear foci. The merged images contain cells with yellow foci (red on a green background)

Table 1 RNF168-deficiency phenotypes, compared with A-T ${ }^{\mathrm{a}}$

\begin{tabular}{|c|c|c|}
\hline Patient $1(15-9 \mathrm{BI})^{26}$ & Patient 2 (RS66) (this study) & Ataxia-telangiectasia \\
\hline $\begin{array}{l}\text { Short stature } \\
\text { No telangiectasia } \\
\text { Mild motor impairment } \\
\text { Learning difficulties } \\
\text { Facial dysmorphism } \\
\text { Hypoimmunoglobulinemia } \\
\text { Cellular radiosensitivity }{ }^{\mathrm{a}}\end{array}$ & $\begin{array}{l}\text { Short stature } \\
\text { Ocular and bronchial telangiectasia } \\
\text { Ataxia, without apraxia or dysarthria } \\
\text { Normal intelligence } \\
\text { Microcephaly } \\
\text { Low serum IgA } \\
\text { Cellular radiosensitivity } \\
\text { Terminal respiratory failure } \\
\text { Elevated serum AFP } \\
\text { Dry skin with fine scaling (xerosis) }\end{array}$ & $\begin{array}{l}\text { Occasional short stature } \\
\text { Telangiectasia } \\
\text { Ataxia, with apraxia and dysarthria } \\
\text { Normal intelligence } \\
\text { Microcephaly (A-T } \text { Fresno variant) }_{\text {Low serum IgA, IgE, IgG2 }} \\
\text { Cellular and clinical radiosensitivity } \\
\text { Terminal respiratory failure } \\
\text { Elevated serum AFP } \\
\text { Cancer susceptibility }\end{array}$ \\
\hline
\end{tabular}

aPotential cancer susceptibility and clinical radiosensitivity of RNF168 deficiency remain to be assessed (see Discussion section)

encompass a large number of immunodeficiency patients with a wide range of clinical manifestations but few defined genetic causes. ${ }^{32}$ As a direct regulator of BRCA1 retention at DSB damage sites, RNF168 may also have a role in breast cancer. ${ }^{33}$ A role for RNF168 in class switch recombination is also suggested by the presence of $\lg A$ deficiency in both RNF168-deficiency patients and the recent studies of Ramachandran et al. ${ }^{12}$ Clinically, the RNF168-deficiency syndrome we describe here is closer to $A-T$ than to the overall milder phenotype of the $15-9 B I$ patient ${ }^{26}$ (Table 1). Whether these clinical differences are attributable solely to the differential severity of RNF168 mutations themselves, or modulated by genetic background of the individual patients and/or interplay with environmental factors, would require identification of additional patients and future studies.
A further implication of our results reflects the specific localization of the RNF168 gene to chromosome 3q29. Deletion of this genomic region is associated with a syndrome of microcephaly, growth retardation short stature, ataxia, chest wall deformities, skin abnormalities and recurrent infections ('3q29 Microdeletion Syndrome'). ${ }^{34}$ These features overlap substantially with those of our new RNF168deficiency patient, RS66, suggesting the possibility that RNF168 deficiency may contribute to the pathogenesis of $3 q 29$ Microdeletion Syndrome. Furthermore, 3q29 is a region where large $(>100 \mathrm{~kb})$ copy number variants (large CNVs) commonly exist, even in normal individuals. These are thought to change gene dosages and be highly penetrant and are reported to be hotspots of human genetic disease, autism and schizophrenia. ${ }^{35-37}$ 
An important next step for understanding the role of RNF168 in disease will be to identify and evaluate additional patients with radiosensitivity, neurodegeneration, cancer, immunodeficiency, microcephaly, autism, growth defects and/or pulmonary fibrosis. As we have demonstrated, the immunocytochemical detection of 53BP1 nuclear foci is a robust functional surrogate screening assay for identifying patients with deficiencies of the chromatin ubiquitin-dependent cascade of DNA repair. The rapid identification of two patients with RNF168 deficiency further suggests that the recently defined pathway may herald a new category of disorders associated with malfunctioning of DNA repair and processing. Last but not least, the fact that RNF168 operates downstream of tumor suppressors, such as ATM and the Mre11/Rad50/Nbs1 complex, and upstream of additional tumor suppressors, such as BRCA1 and 53BP1, ${ }^{16}$ raises the issue of whether the RNF168-deficiency syndrome may include a predisposition to cancer. Given that cancer incidence among patients suffering from the A-T syndrome, is approximately $30 \%$ (see also Table 1), it is important that this question be addressed as well for the RNF168-deficiency syndrome, which will require clinical follow-up and family history studies of a larger number of patients. While there was only limited information about the family history of the RS66 patient, the father of the other patient with RNF168 deficiency ${ }^{7}$ developed chronic B-cell lymphocytic leukemia, a malignancy that also occurs in A-T patients. Overall, considering the role of the DSB signaling, repair and checkpoint machinery as an intrinsic barrier against activated oncogenes and tumor progression, ${ }^{38-40}$ it is reasonable to speculate that RNF168 itself represents a plausible candidate for a potential tumor suppressor.

\section{Materials and Methods}

All studies were conducted in accordance with Institutional Review Board requirements and appropriate approvals were obtained.

Colony survival assay. We assessed the radiosensitivity of LCLs using CSA, as previously described. ${ }^{20}$ Briefly, after the LCLs were exposed to varying doses of IR from 0 to $2 \mathrm{~Gy}$, they were incubated at $37^{\circ} \mathrm{C}$ for $10-14$ days, depending on cell growth. After MTT staining, each well was scored for the presence-absence of colonies of $>32$ cells (five divisions) and colony SFs (\%CSF) were calculated. On the basis of an earlier study comparing 104 bona fide A-T cell lines with 29 wild-type cells, the \%CSF was interpreted as 'radiosensitive' if below $21 \%$ SF (13 $\pm 7 \%$ ) for $1 \mathrm{~Gy}$ exposure. Radiosensitive and radionormal controls were included in each experiment.

Immunocytochemistry and $\gamma$-H2AX foci kinetics. $L C L$ lines from RS66 and controls were exposed to 3 Gy radiation to induce double-strand DNA breaks. After 1-h incubation, cells were fixed in $4 \%$ phosphate-buffered formalin, permeabilized with $0.5 \%$ Triton $\mathrm{X}-100$ and immunostained with antibodies to $\gamma$-H2AX (Upstate, Billerica, MA, USA; \#05-636), MDC1 (Abcam, Cambridge, UK; \#ab11171), 53BP1 (Santa Cruz, CA, USA; \#sc-22760) or BRCA1 (Santa Cruz; \#sc-6954), as specified in Figure legends, and followed with Alexa Fluor 488 and Alexa Fluor 568 secondary antibodies (Invitrogen, Carlsbad, CA, USA). Slides were mounted in DAPI Vectashield (Vector Laboratories, Burlingame, CA, USA) and analyzed by a confocal fluorescence microscopy as described. ${ }^{6,13}$ To assess the kinetics of $\gamma-\mathrm{H} 2 \mathrm{AX}$ foci, exponentially growing cells were irradiated with a dose of $1 \mathrm{~Gy}$, and samples for immunocytochemical estimation were taken at times 0 (preIR state), 1 and $24 \mathrm{~h}$ post radiation. At least 300 cells were counted per sample, and cells with $>4 \gamma-\mathrm{H} 2 \mathrm{AX}$ foci were regarded as positive.
Western blotting. Protein extracts were resolved by 7.5\% SDS-PAGE, transferred to the nitrocellulose or PVDF membranes, blocked with $5 \%$ evaporated milk (Bio-Rad, Hercules, CA, USA), and probed with the following antibodies: mouse monoclonal antibody to RNF168 (DCS-402; generated for the purpose of this study by standard hybridoma technology); rabbit polyclonal antibodies to RNF168 (gift from Drs. Daniel Durocher and Stephanie Panier), ATM (Abcam; \#ab31842), sheep polyclonal antibody to MDC1 (gift from Dr. Stephen Jackson), $\mathrm{RNF8}^{7}$ and mouse monoclonal antibody to MCM7 (DCS-141). ${ }^{6}$

Radioresistant DNA synthesis. LCLs were incubated with ${ }^{14} \mathrm{C}$ thymidine for $24 \mathrm{~h}$ to establish background synthesis of DNA. The medium was replaced with fresh media, and the cells were exposed to varying doses of $\operatorname{IR}(0,2,5,10$ and 25 Gy). The cells were returned to the incubator for $60 \mathrm{~min}$ and pulse labeled in medium containing tritiated thymidine $\mathrm{H}^{3} \mathrm{~T}$ for an additional $60 \mathrm{~min}$. The samples were harvested and counted in a Packard 2900TR scintillation counter (Packard, Mt Waverley, VIC, Australia). The ratio of incorporated $\mathrm{H}^{3} \mathrm{~T}$ to ${ }^{14} \mathrm{C}$ was used as a measure of the amount of DNA synthesis after IR exposure. Experiments were performed in triplicate.

SMC1 phosphorylation kinetics. Nuclear extracts from 5 to 10 million RS66, WT or A-T LCLs were prepared following the manufacturer's protocol (NE-PER Nuclear and Cytoplasmic Extraction Reagents, Pierce, Rockford, IL, USA). LCLs were harvested 1,4 and $24 \mathrm{~h}$ after $10 \mathrm{~Gy}$ or mock treatment. In all, $25 \mathrm{~g}$ of nuclear lysate were electrophoresed on a 7.5\% SDS-polyacrylamide gel (PAGE), transferred onto PVDF membrane (Bio-Rad), and blocked with $5 \%$ dry milk (Bio-Rad). Blots were incubated overnight at $4{ }^{\circ} \mathrm{C}$ with the following antibodies: anti-SMC1s966, (Novus, Littleton, $\mathrm{CO}$, USA), and SMC1, (Novus). An HRP-conjugated anti-rabbit or anti-mouse secondary antibody was incubated at room temperature for $40 \mathrm{~min}$ for detection by enhanced chemiluminescence (Amersham Pharmacia, Piscataway, NJ, USA).

DNA sequencing. Genomic DNA was isolated from RS66 and PCR amplified using six primer sets (forward and reverse) for each exon. After the PCR amplification, the products were visualized using agarose gel electrophoresis and purified (Qiagen PCR purification kit; Qiagen, Valencia, CA, USA). The purified PCR products were used to sequence each exon, in addition to 200-bp upstream and downstream of the flanking regions. DNA sequences were compared with wild-type sequences using blast 2 program from the NCBI website (http:// blast.ncbi.nlm.nih.gov/Blast.cgi).

\section{Conflict of interest}

The authors declare no conflict of interest.

Acknowledgements. Dr. Nermin Capan provided pulmonary care for the patient at the Atatürk Chest Disease and Chest Surgery, Education and Research Hospital, Ankara, Turkey. We thank Drs. Daniel Durocher, Stephanie Panier and Stephen Jackson for reagents. CD, JB, CL and JL were supported by the Danish Cancer Society, the Danish National Research Foundation, the Lundbeck Foundation (R13-A1287 and R44-A4400), the Czech Ministry of Education (MSM6198959216) and the European Community (projects: GENICA, Infla-Care and CZ.1.05/2.1.00/01.0030). SSD, KN, KP, SAN and RAG were supported by USPHS grants (Al 067769, NS052528), the A-T Ease Foundation, the A-T Medical Research Foundation, the Joseph Drown Foundation, and by a fellowship (SAN) from the University of California Toxic Substances Research and Teaching Program.

1. Westbrook AM, Schiestl RH. Atm-deficient mice exhibit increased sensitivity to dextran sulfate sodium-induced colitis characterized by elevated DNA damage and persistent immune activation. Cancer Res 2010; 70: 1875-1884.

2. Wood RD, Mitchell M, Sgouros J, Lindahl T. Human DNA repair genes. Science 2001; 291: 1284-1289.

3. Jackson SP, Bartek J. The DNA damage response in human biology and disease. Nature 2009; 461: 1071-1078.

4. Kastan BM, Bartek J. Cell-cycle checkpoints and cancer. Nature 2004; 432: 316-323.

5. Nahas SA, Gatti RA. DNA double strand break repair defects, primary immunodeficiency disorders, and 'radiosensitivity'. Curr Opin Allergy Clin Immunol 2009; 9: 510-516.

6. Doil C, Mailand N, Bekker-Jensen S, Menard P, Larsen DH, Pepperkok R et al. RNF168 binds and amplifies ubiquitin conjugates on damaged chromosomes to allow accumulation of repair proteins. Cell 2009; 136: 435-446. 
7. Stewart G, Panier S, Townsend K, Al-Hakim AK, Kolas NK, Miller ES et al. The RIDDLE syndrome protein mediates a ubiquitin-dependent signaling cascade at sites of DNA damage. Cell 2009; 136: 420-434.

8. Al-Hakim A, Escribano-Diaz C, Landry MC, O'Donnell L, Panier S, Szilard RK et al. The ubiquitous role of ubiquitin in the DNA damage response. DNA Repair 2010; 9: 1229-1240.

9. Bartek J, Lukas J. DNA damage checkpoints: from initiation to recovery or adaptation. Curr Opin Cell Biol 2007; 19: 238-245.

10. Mailand N, Bekker-Jensen S, Faustrup H, Melander F, Bartek J, Lukas C et al. RNF8 ubiquitylates histones at DNA double-strand breaks and promotes assembly of repair proteins. Cell 2007; 131: 887-900.

11. Kolas NK, Chapman JR, Nakada S, Ylanko J, Chahwan R, Sweeney FD et al. Orchestration of the DNA-damage response by the RNF8 ubiquitin ligase. Science 2007; 318: $1637-1640$

12. Ramachandran S, Chahwan R, Nepal RM, Frieder D, Panier S, Roa S et al. The RNF8/ RNF168 ubiquitin ligase cascade facilitates class switch recombination. Proc Natl Acad Sci USA 2010; 107: 809-814.

13. Bekker-Jensen S, Rendtlew Danielsen J, Fugger K, Gromova I, Nerstedt A, Lukas C et al. HERC2 coordinates ubiquitin-dependent assembly of DNA repair factors on damaged chromosomes. Nat Cell Biol 2010; 12: 80-86.

14. Galanty Y, Belotserkovskaya R, Coates J, Polo S, Miller KM, Jackson SP et al. Mammalian SUMO E3-ligases PIAS1 and PIAS4 promote responses to DNA double-strand breaks. Nature 2009; 462: 935-939.

15. Morris JR, Boutell C, Keppler M, Densham R, Weekes D, Alamshah A et al. The SUMO modification pathway is involved in the BRCA1 response to genotoxic stress. Nature 2009; 462: $886-890$

16. Bartek J, Hodny Z. SUMO boosts the DNA damage response barrier against cancer. Cancer Cell 2010; 17: 9-11.

17. Shiloh $Y$, Kastan MB. ATM: genome stability, neuronal development and cancer cross paths. Adv Cancer Res 2001; 83: 209-254.

18. Chun HH, Gatti RA. Ataxia-telangiectasia, an evolving phenotype. DNA Repair 2004; 3 1187-1196.

19. Ishiguro T, Taketa K, Gatti RA. Tissue of origin of elevated alpha-fetoprotein in ataxiatelangiectasia. Dis Markers 1986; 4: 293-297.

20. Sun X, Becker-Catania SG, Chun HH, Hwang MJ, Huo Y, Wang $Z$ et al. Early diagnosis of ataxia-telangiectasia using radiosensitivity testing. J Pediatr 2002; 140: 724-731.

21. Pollard J, Gatti RA. Clinical radiation sensitivity with DNA repair disorders: an overview. Int J Radiat Oncol Biol Phys 2009; 74: 1323-1331.

22. Ambrose M, Goldstine JV, Gatti RA. Intrinsic mitochondrial dysfunction in ATM-deficient lymphoblastoid cells. Hum Mol Genet 2007; 16: 2154-2164.

23. Perrault RB, Wang $H$, Wang $M$, Rosidi B, lliakis G. Backup pathways of NHEJ are suppressed by DNA-PK. J Cell Biol 2004; 92: 781-794.
24. Schultz LB, Chehab NH, Malikzay A, Halazonetis TD. P53 binding protein 1 (53BP1) is an early participant in the cellular response to DNA double strand breaks. J Cell Biol 2000; 151: $1381-1390$.

25. DiTullio Jr RA, Mochan TA, Venere M, Bartkova J, Sehested M, Bartek J. 53BP1 functions in an ATM-dependent checkpoint pathway that is constitutively activated in human cancer Nat Cell Biol 2002; 4: 998-1002.

26. Stewart GS, Stankovic T, Byrd PJ, Wechsler T, Miller ES, Huissoon A et al. RIDDLE immunodeficiency syndrome is linked to defects in 53BP1-mediated DNA damage signaling. Proc Natl Acad Sci USA 2007; 104: 16910-16915.

27. Asaka T, Yokoji H, Ito J, Yamaguchi K, Matsushima A. Autosomal recessive ataxia with peripheral neuropathy and elevated AFP: novel mutations in SETX. Neurology 2006; 66 1580-1581.

28. Rigby RE, Leitch A, Jackson A. Nucleic acid mediated inflammatory diseases. Bioessays 2008; 30: 833-842.

29. Lawson WE, Loyd JE. The genetic approach in pulmonary fibrosis: can it provide clues to this complex disease? Proc Am Thorac Soc 2006; 3: 345-349.

30. Scotton $\mathrm{C}$, Chambers R. Molecular targets in pulmonary fibrosis the myofibroblast in focus. Chest 2007; 132: 1311-1321.

31. Tangsinmankong N, Wayne AS, Howenstine MS, Washington KR, Langston C, Gatti RA et al. Lymphocytic interstitial pneumonitis, elevated $\operatorname{lgM}$, and hepatosplenomegaly in ataxia-telangiectasia. J Pediat 2001; 138: 939-941.

32. Bonilla FA, Geha RS. Common variable immunodeficiency. Pediatr Res 2009; 65: 13R-19R.

33. Tan $D$, Marchiò $C$, Reis-Filho JS. Hereditary breast cancer: from molecular pathology to tailored therapies. J Clin Pathol 2008; 61: 1073-1082.

34. Willatt L, Cox J, Barber J, Cabanas ED, Collins A, Donnai D. Microdeletion syndrome: clinical and molecular characterization of a new syndrome. Am J Hum Genet 2005; 77 154-160.

35. Itsara A, Cooper GM, Baker C, Girirajan S, Li J, Absheer D et al. Population analysis of large copy number variants and hotspots of human genetic disease. Am J Hum Genet 2009; 84: 148-161.

36. Mulle JG, Dodd AF, McGrath JA, Wolyniec PS, Mitchell AA, Shetty AC et al. Microdeletions of 3q29 confer high risk for schizophrenia. Am J Hum Genet 2010; 87: 229-236.

37. Quintero-Rivera F, Sharifi-Hannauer P, Martinez-Agosto JA. Autistic and psychiatric findings associated with the 3q29 microdeletion syndrome: case report and review. Am J Med Genet A 2010; 152: 2459-2467.

38. Gorgoulis VG, Vassiliou LV, Karakaidos P, Zacharatos P, Kotsinas A, Liloglou T et al Activation of the DNA damage checkpoint and genomic instability in human precancerous lesions. Nature 2005; 434: 907-913.

39. Bartkova J, Horejsi Z, Koed K, Kramer A, Tort F, Zieger K et al. DNA damage response as a candidate anti-cancer barrier in early human tumorigenesis. Nature 2005; 434: 864-870.

40. Halazonetis TD, Gorgoulis VG, Bartek J. An oncogene-induced DNA damage model for cancer development. Science 2008; 319: 1352-1355. 